\title{
All-optical OFDM demultiplexing with optical partial Fourier transform and coherent sampling
}

Geng, Zihan; Kong, Deming; Corcoran, Bill; Guan, Pengyu; Da Ros, Francesco; Porto da Silva, Edson; Oxenløwe, Leif Katsuo; Lowery, Arthur James

\section{Published in:}

Optics Letters

Link to article, DOI:

10.1364/OL.44.000443

Publication date:

2019

Document Version

Publisher's PDF, also known as Version of record

Link back to DTU Orbit

Citation $(A P A)$ :

Geng, Z., Kong, D., Corcoran, B., Guan, P., Da Ros, F., Porto da Silva, E., Oxenløwe, L. K., \& Lowery, A. J. (2019). All-optical OFDM demultiplexing with optical partial Fourier transform and coherent sampling. Optics Letters, 44(2), 443-446. https://doi.org/10.1364/OL.44.000443

\section{General rights}

Copyright and moral rights for the publications made accessible in the public portal are retained by the authors and/or other copyright owners and it is a condition of accessing publications that users recognise and abide by the legal requirements associated with these rights.

- Users may download and print one copy of any publication from the public portal for the purpose of private study or research.

- You may not further distribute the material or use it for any profit-making activity or commercial gain

- You may freely distribute the URL identifying the publication in the public portal 


\title{
All-optical OFDM demultiplexing with optical partial Fourier transform and coherent sampling
}

\author{
Zihan Geng, ${ }^{1,2}$ Deming Kong, ${ }^{1,2,4, *}$ (1) Bill Corcoran, ${ }^{1,2}$ (1) Pengyu Guan, ${ }^{3}$ Francesco Da Ros, ${ }^{3}$ (1) \\ Edson Porto da Silva, ${ }^{3,5}$ (1) Leif Katsuo Oxenløwe, ${ }^{2,3}$ and Arthur James Lowery ${ }^{1,2}$ \\ ${ }^{1}$ Electro-Photonics Laboratory, Department of Electrical and Computer Systems Engineering, Monash University, Clayton, VIC 3800 , Australia \\ ${ }^{2}$ Centre for Ultrahigh-Bandwidth Devices for Optical Systems (CUDOS), Australia \\ ${ }^{3}$ DTU Fotonik, Technical University of Denmark (DTU), DK-2800 Kgs. Lyngby, Denmark \\ ${ }^{4}$ Current address: DTU Fotonik, Technical University of Denmark (DTU), DK-2800 Kgs. Lyngby, Denmark \\ ${ }^{5}$ Current address: Department of Electrical Engineering, Federal University of Campina Grande (UFCG), 58429-900, Campina Grande, Brazil \\ ${ }^{*}$ Corresponding author: dmkon@fotonik.dtu.dk
}

Received 8 October 2018; revised 11 December 2018; accepted 17 December 2018; posted 18 December 2018 (Doc. ID 346162);

published 15 January 2019

\begin{abstract}
We propose a novel scheme with a "time-lens"-based partial optical Fourier transform (OFT) and coherent sampling for high-speed complex orthogonal frequency-division multiplexing (OFDM) signal detection. Compared with all-optical OFDM demultiplexing with a matched optical filter, our proposed method replaces specialized optical filters with commercially available equipment, which relaxes stringent manufacturing and operational requirements. Our simulation shows that even with a partial OFT, theoretically, close to inter-channel interference-free performance is possible. In addition, we performed a proof-of-concept experiment of $16 \times 10$ Gbaud quadrature phase-shift keying (QPSK) all-optical OFDM detection, with all the bit error rates far below the 7\% hard-overhead forward error correction limit. (C) 2019 Optical Society of America
\end{abstract}

https://doi.org/10.1364/OL.44.000443

All-optical orthogonal frequency-division multiplexing (AOOFDM) enables ultra-high-speed optical communication with high spectral efficiency. Broadband AO-OFDM can be generated by multiplexing several sinc-shaped subcarriers orthogonally [1]. However, the reception of such broadband, tightly spaced signals can be challenging. Theoretically, an OFDM signal can be decoded with a digital fast Fourier transform but, in this case, the maximum capacity is limited by the electrical bandwidths of coherent receivers, the bandwidths of analogto-digital converters and the speed of digital signal processing (DSP) [2]. By contrast, all-optical OFDM demultiplexers can detect ultra-high-speed, broadband OFDM signals [3-9]. In general, $\mathrm{AO}-\mathrm{OFDM}$ demultiplexers perform an optical discrete Fourier transform, which acts as a matched filter [3,4]. Alloptical demultiplexers based on cascaded optical delay interferometers [5], arrayed-waveguide gratings [6], passive planar hybrids [7], and multimode interference [8] have been reported. However, OFDM demultiplexing based on optical matched sinc filters requires precise matching between the signal and the filter in terms of amplitude, phase, and central frequency $[2,6,10]$. It also requires phase stabilization. These factors largely confine the flexibility and scalability of such devices. Furthermore, practically optical sinc filters are spectrally truncated and so significant inter-channel interference (ICI) may be introduced by imperfect matched filtering, which in photonic integrated circuits can also arise naturally from manufacturing variability [11]. For wide-band OFDM signals, precise optical sampling is needed before photodetection $[2,5]$.

Optical time-lenses are capable of ultra-high-speed Fourier transformation of multiple channels [12]. Recently, two AO-OFDM demultiplexing schemes based on time-lenses were proposed by Palushani [9] and Guan [13]. One method is to magnify the spectra of OFDM signals with a spectral imaging time-lens, which allows optical low-pass filters to extract each subcarrier [9]. This method avoids optical sampling, but it will always introduce interference from the spectrally overlapped subcarriers. Another method is to convert the AO-OFDM signal to a Nyquist optical time-division multiplexed (N-OTDM) signal using a partial OFT time-lens [13], then time-demultiplex the N-OTDM signal by optical sampling using a nonlinear optical loop mirror and Gaussian pulses.

In this Letter, we propose a novel AO-OFDM demultiplexing method with a time-lens-based OFDM-to-N-OTDM conversion $[14,15]$ and optical sampling in a coherent receiver. To the best of our knowledge, this is the first time that time-lenses and coherent sampling for AO-OFDM demultiplexing have been combined. This methodology enables the detection of high-speed phase-encoded OFDM signals with low-bandwidth coherent receivers.

Figure 1 shows the principle of our proposed ICI-free AO-OFDM receiver. Here the AO-OFDM signal is Fourier transformed to a N-OTDM signal by a time-lens. After the conversion, the ICI of the OFDM signal is mapped to the inter-symbol-interference (ISI) of a N-OTDM signal. Instead of performing a complete OFT with dispersion-chirp-dispersion (D-K-D) or chirp-dispersion-chirp (K-D-K) structure, we selected 


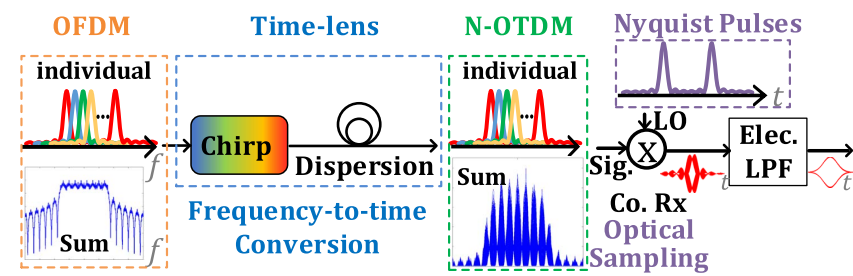

Fig. 1. Principle of our proposed AO-OFDM receiver.

a partial OFT structure for several reasons. First, the D-K-D structure requires guard intervals between OFDM symbols to avoid the overlap due to the pulse broadening at the first dispersion stage. Secondly, the K-D-K structure avoids the overlapping problem, but it requires two chirp stages. Thirdly, the result of optical sampling is primarily affected by the time domain waveform, so a frequency-to-time mapping with a chirpdispersion (K-D) structure is sufficient.

Ideally, the chirp should be linear and periodic with a repetition rate equaling the baud rate of the signal $\mathrm{BR}_{\text {sig }}$ for symbol-by-symbol OFT. The chirp rate of the time-lens is represented as $K$. The accumulated dispersion $D$ of a dispersive fiber is given by the group-velocity dispersion parameter $\beta_{2}$ multiplied by the length of the fiber $L$. The OFT condition is $D=1 / K$. If we represent the $3 \mathrm{~dB}$ bandwidth of the OFDM signal with $\mathrm{BW}_{\text {sig }}$, after the OFT, the corresponding temporal width is given by $\mathrm{BW}_{\text {sig }} / K$. Similarly, given an OFDM channel spacing of $\mathrm{CS}_{\text {sig }}$, the time interval between the converted tributaries is given by $\mathrm{CS}_{\text {sig }} / K$. There are two design constraints on the chirp rate. First, the chirp rate $K$ should be faster than $\mathrm{BW}_{\text {sig }} \times \mathrm{BR}_{\text {sig }}$ to avoid overlap. Secondly, the chirp rate $K$ should be an integer multiple of $\mathrm{CS}_{\text {sig }} \times \mathrm{BR}_{\text {sig }}$ to align the ISI-free points of the sinc pulses generated from successive OFDM symbols.

In the sampling stage, the coherent sampler performs optical sampling and coherent detection at the same time, which avoids the need for a separate sampling stage [16]. The local oscillator (LO) used for sampling is a sinc pulse train. According to Refs. $[16,17]$, optical sampling a N-OTDM signal with sinc pulses outperforms sampling using Gaussian pulses.

Ideal simulations using VPItransmissionMaker are performed to estimate the theoretical penalty of our proposed method. In the simulation, a single-channel 10 GBaud quadrature phaseshift keying (QPSK) and a zero-guard-band $16 \times 10$ Gbaud QPSK OFDM signal are processed by a time-lens and coherent sampling. The time-lenses are composed of linear-chirp modules and matched-dispersion modules. To achieve an N-OTDM-like signal with a 5 ps tributary spacing, the chirp rate is set to $-0.0126 \mathrm{ps}^{-2}$. Then the N-OTDM-like signal is sampled by sinc-shaped pulses with a 1.6 ps $3 \mathrm{~dB}$ FWHM width. The 5 and $1.6 \mathrm{ps}$ widths are chosen for consistency between our experiments and simulations. In terms of the 16-channel OFDM signal, the 7th channel is measured in the simulation.

In Fig. 2, a comparison of BERs between the single-channel QPSK back-to-back (B2B) and the single-channel QPSK processed by a K-D time-lens shows a negligible difference, indicating there is no penalty from the K-D time-lens. In addition, there is negligible difference between the K-D and the K-D-K time-lens-based 16-channel OFDM detection, proving that one chirp stage is sufficient for this application. Compared with the B2B case, the K-D time-lens-based OFDM detection only

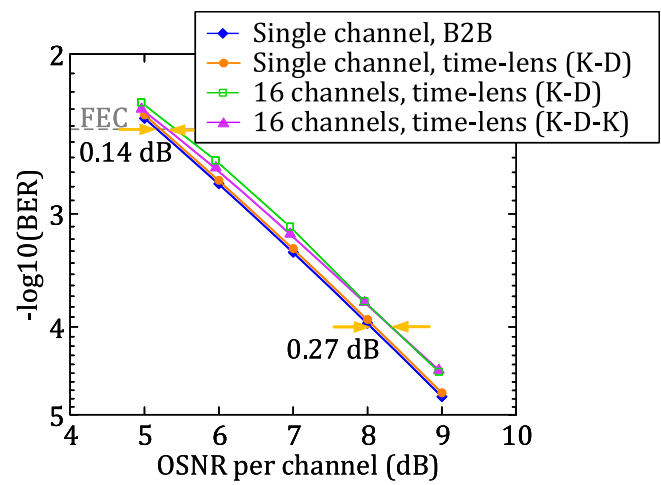

Fig. 2. BER comparison of time-lens-based $16 \times 10$ Gbaud QPSK OFDM against the B2B 10 GBaud QPSK detection.

has $0.14 \mathrm{~dB}$ penalty at BER $=3.8 \times 10^{-3}$ and $0.27 \mathrm{~dB}$ penalty at $\mathrm{BER}=10^{-4}$. This small penalty is introduced by the sampling process, where the sampling pulses are not sufficiently short to completely eradicate the ISI. A narrower sampling pulse would result in less ISI, but it may introduce a penalty due to the power loss and noise in the system.

Figure 3 shows the experimental setup for $16 \times 10$ Gbaud QPSK AO-OFDM generation and reception. An optical frequency comb with $10 \mathrm{GHz}$ spacing is generated by a mode-locked laser (MLL) and then broadened in a $400 \mathrm{~m}$ dispersion-flattened highly nonlinear fiber (DF-HNLF) $\left(\gamma=10.7 \mathrm{~W}^{-1} \mathrm{~km}^{-1}, \beta_{2}=\right.$ $-0.446 \mathrm{ps}^{2} / \mathrm{km}$, and $\beta_{3}=0.0057 \mathrm{ps}^{3} / \mathrm{km}$, at $\left.1550 \mathrm{~nm}\right)$. The broadened comb is filtered at three different central wavelengths and diverted to three output ports of a wavelength selective switch (WSS1), for the generation of the AO-OFDM signal, the chirped pump, and the LO, respectively.

In the $\mathrm{AO}-\mathrm{OFDM}$ generation sub-system, a rectangular bandpass filter with a $1560.96 \mathrm{~nm}$ central wavelength and a $500 \mathrm{GHz}$ pass bandwidth are set at Port 1 of WSS1. The pulse train at Output 1 is time aligned and polarization aligned to the IQ modulator by a time delay and a polarization controller (PC). The output of the IQ modulator is a 10 GBaud QPSK signal with a $500 \mathrm{GHz}$ bandwidth and flat-top spectra, and it is equally divided into four paths. The signals in the four paths are decorrelated by dispersion shifted fibers of 0,125 , and $250 \mathrm{~m}$, and $1 \mathrm{~km}$. The symbols are time-aligned with fine delay lines. WSS2 performs optical inverse Fourier transforms by applying four filters [1]. Each filter passes and sinc-shapes four subcarriers separated by $80 \mathrm{GHz}$. The filter is shifted by $20 \mathrm{GHz}$ between every two adjacent ports. The subcarrier spacing of the OFDM signal is set to $20 \mathrm{GHz}$ for a $50 \%$ guard interval between symbols. The guard interval is required to avoid the temporal overlap of the signal and the rising or falling edges of the chirped pump in the FWM process. In addition, considering the $10 \mathrm{GHz}$ optical transfer function of WSS2, the bandwidth of each OFDM subcarrier was designed as $20 \mathrm{GHz}$ to avoid the filter distortion. In order to evaluate the time-lens-based receiver performance, noise generated by an erbium-doped fiber amplifier (EDFA) is coupled with the AO-OFDM signal at the input of the time lens, where the optical signal-to-noise ratio (OSNR) is measured.

At the chirped pump generation stage, the bandpass filter at Port 2 of WSS1 is centered at $1550.39 \mathrm{~nm}$ with a $180 \mathrm{GHz}$ bandwidth. To achieve the desired chirp rate, the accumulated dispersion experienced by the pump is $79.6 \mathrm{ps}^{2}$, which is 


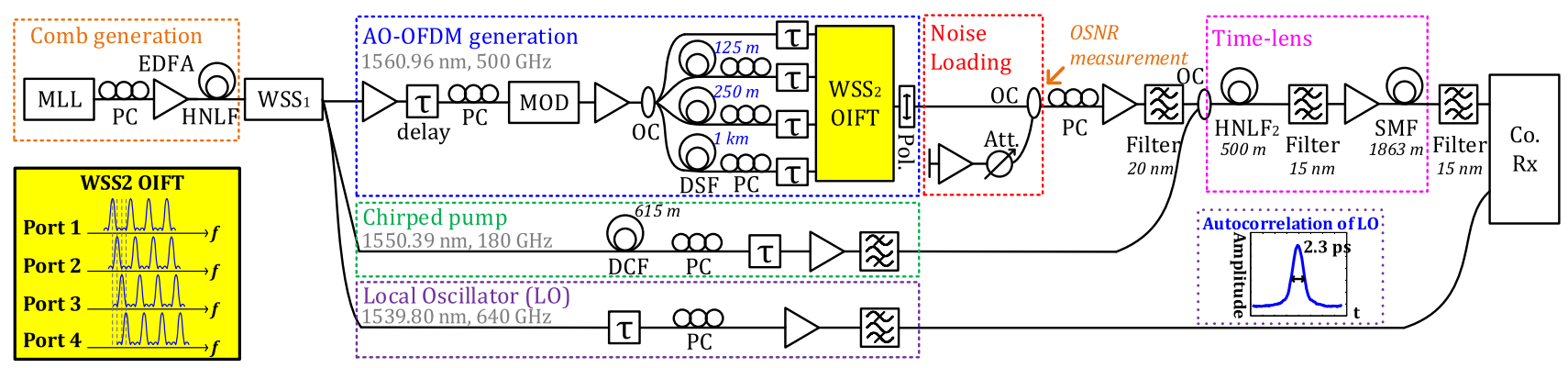

Fig. 3. Experimental setup of time-lens and optical sampling-based AO-OFDM demultiplexing.

slightly less than the number calculated from the Fraunhofer condition [18] to avoid pulse overlap. The $615 \mathrm{~m}$ dispersion compensation fiber chirps the pump and broadens the pump pulses in the time domain. The chirped pump is amplified by a high-power EDFA followed by a bandpass filter.

At the time-lens stage, the signal power and the pump power coupled into HNLF2 are -2.5 and $20.5 \mathrm{dBm}$, respectively. The OFDM signal and the chirped pump generate a new frequency component called the "idler," which is a N-OTDM signal at $1539.8 \mathrm{~nm}$ by four-wave mixing in HNLF2. The idler is extracted by a bandpass filter and dispersed by an $1863 \mathrm{~m}$ standard SMF. The accumulated dispersion of this SMF is $-39.8 \mathrm{ps}^{2}$, which corresponds to a chirp rate of $-0.0251 \mathrm{ps}^{-2}$.

At the sampling stage, the width of the LO measured by the auto-correlator is $2.3 \mathrm{ps}$, which corresponds to a $1.6 \mathrm{ps} 3 \mathrm{~dB}$ pulse width. The central frequency of the $\mathrm{LO}$ is equal to the central frequency of the idler. The subcarriers are selected by tuning the time delay of the $\mathrm{LO}$. The signal and the LO powers at the input of the optical modulation analyzer (OMA) are 4.5 and $0.5 \mathrm{dBm}$, respectively. The OMA includes a digital sampling scope, a coherent receiver and a semiconductor optical amplifier to amplify the LO. The band limitation of the coherent receiver acts as an electrical low-pass filter. DSP algorithms have been utilized to recover the demultiplexed channel.

Figure 4 shows the waveform and spectra of the chirped pump and the AO-OFDM signal. The waveform is detected by a $40 \mathrm{GHz}$ photodetector and a $50 \mathrm{GHz}$ bandwidth equivalenttime sampling oscilloscope. In Fig. 4(a), the chirped pump pulses have amplitude fluctuations due to the insufficiently broad pump bandwidth $(180 \mathrm{GHz})$. Figure 4(c) shows the corresponding simulation results, without experimental bandwidth limitations, of the pump waveform, where amplitude fluctuations can also be observed. The fluctuations on the amplitude result in the uneven amplitude of the converted signal. In addition, as the chirp is not perfectly linear, different tributaries can overlap after the time-lens, causing ISI. According to the Fraunhofer requirement [18] and the simulation result in Fig. 4(d), the bandwidth of the pump has to be sufficiently large (i.e., $1.8 \mathrm{THz}$ ), so that after certain dispersion, closeto-linear chirp and rectangular waveforms can be achieved. However, a larger pump bandwidth results in a narrower required LO pulse width and a more stringent requirement on the temporal alignment and time jitter. There may be an optimal pump bandwidth between $180 \mathrm{GHz}$ and $1.8 \mathrm{THz}$, which may be interesting to investigate in the future. In Fig. 4(b), we show the experimental spectrum of an AOOFDM signal with a flat top and several sidelobes on each side.
Figure 5(a) shows the output spectrum of HNLF2. Figure 5(b) shows the bit error rates (BERs) of all 16 demultiplexed AO-OFDM channels at $18 \mathrm{~dB}$ OSNR per channel. We assume that the system will be running with a $7 \%$ overhead forward error correction (FEC) code to bring a pre-FEC measured BER down from $3.8 \times 10^{-3}$ to an error-free level of $1 \times 10^{-15}$. The maximum measured BER is $4.5 \times 10^{-5}$. The minimum reliably measured BER was $1.6 \times 10^{-6}$, based on measuring an average of 10 errors. Channels $7,9,11,13$, and 15 were below this threshold in the experiment; thus, their BERs are presented by arrows in Fig. 5(b). There are some differences in the BERs of different channels. This may be attributed to the aforementioned amplitude and chirp fluctuations of the pump.

Figure 5(c) shows the performance of the 7th, 8 th, and 16th OFDM channels measured from the experiment and the corresponding simulation result of the 7th channel. In the simulation, the chirp, dispersion, and AO-OFDM generation are

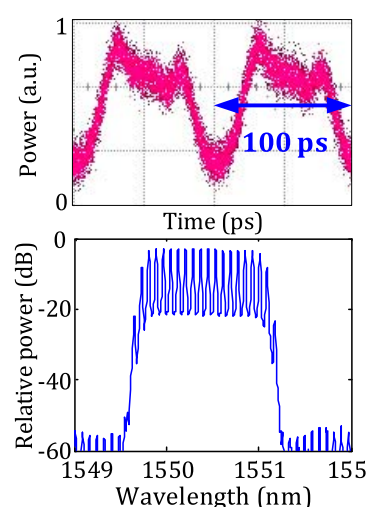

(a)

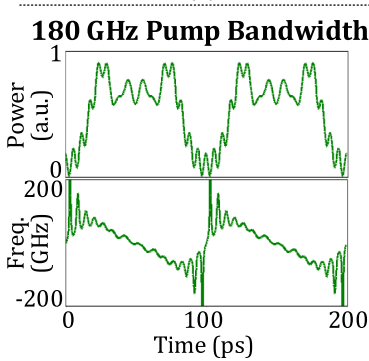

(c)

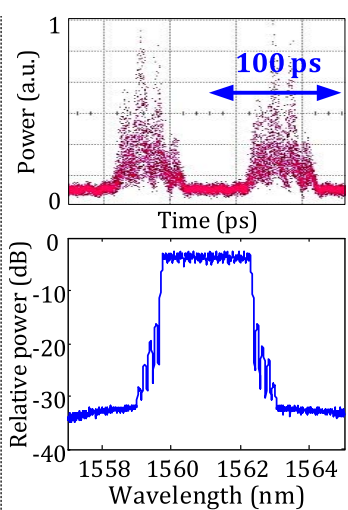

(b)

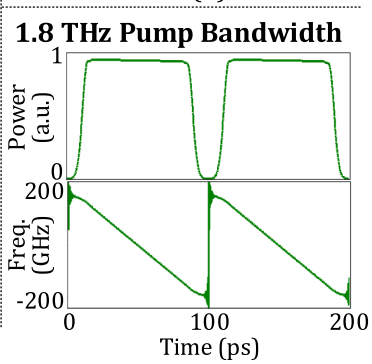

(d)
Fig. 4. Experimental waveforms and spectra of (a) chirped pump and (b) AO-OFDM signals. Simulated waveforms and frequencies of a chirped pump with (c) $180 \mathrm{GHz}$ or (d) $1.8 \mathrm{THz}$ initial bandwidth. 


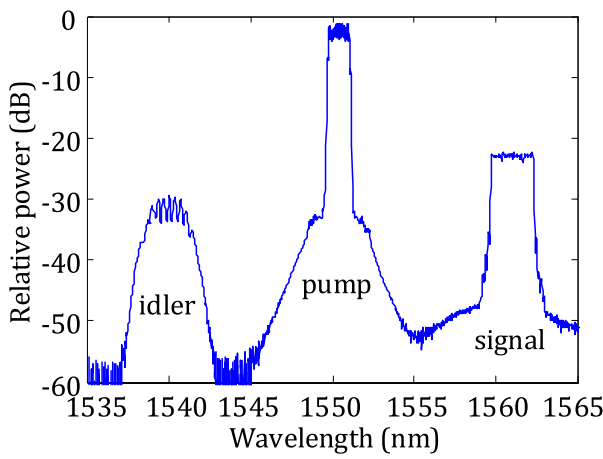

(a)

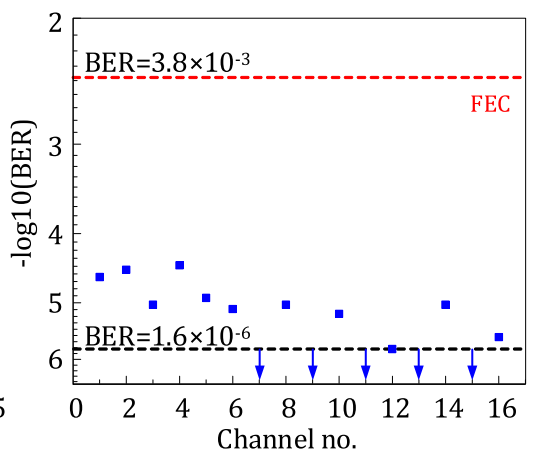

(b)

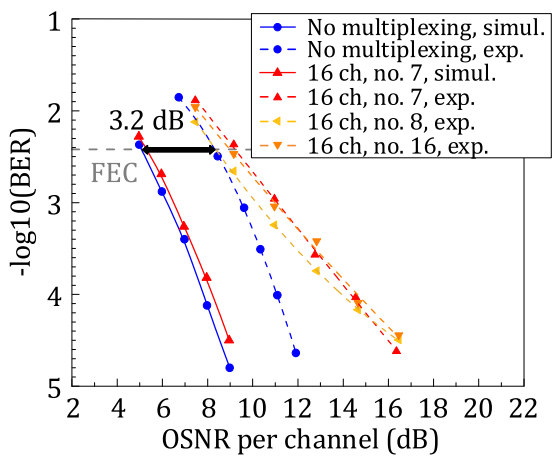

(c)

Fig. 5. (a) Output spectrum of HNLF2. (b) The performances of the16 subcarriers at $18 \mathrm{~dB}$ OSNR per channel. (c) BER versus OSNR per channel plot of the 160 GBd OFDM signal and the 40 GBd coarse WDM signal.

ideal. Comparing the simulation and the experimental results of the 7 th channel, there is about a $3.7 \mathrm{~dB}$ difference. To investigate the source of the difference, a close-to-ICI-free case was measured by blocking Ports 2 to 4 of WSS2. Therefore, only Port 1 was retained, and all of the four sinc-shaped subcarriers out of Port 1 carry the same information. In this case, there is no multiplexing of different information, so it is labeled as "No multiplexing" in Fig. 5(c). Comparing the measured and simulated "No multiplexing" results, there is a $3.2 \mathrm{~dB}$ difference at the FEC limit. The measured OSNR may be slightly different from the actual OSNR, because the narrow filtering in WSS2 does not allow a precise estimate of the in-band noise. In addition, the undesired nonlinear mixing in the highly nonlinear fiber may introduce distortion [19].

In the experiment, the difference between the OFDM channels and "No multiplexing" case increases from less than $1 \mathrm{~dB}$ at $\mathrm{BER}=3.8 \times 10^{-3}$ to about $4 \mathrm{~dB}$ at $\mathrm{BER}=10^{-4}$, which may be due to the noise in the system and the interference among channels. The interference can originate from the imperfections in the time-lens [20], particularly the pump-imposed chirp, or imperfect definition and crosstalk of sub-carrier shaping through WSS2 [21,22].

In conclusion, we have proposed a novel AO-OFDM demultiplexing scheme based on time-lenses and coherent optical sampling. Our method avoids the problems of arbitrary waveform generator-based OFDM demultiplexers such as manufacturing variability. The simulations show that theoretically close to ISI-free performance is possible to achieve. We also experimentally demonstrated the demultiplexing of a 320 Gbps AO-OFDM signal with BERs below the 7\% hard-FEC limit. It may be interesting to use temporal matched sampling $[11,23,24]$, together with time-lens-based OFT to detect high-speed OFDM signals in the future.

Funding. Australian Research Council (ARC) (CE110001018); ARC Laureate Fellowship (FL130100041); National Natural Science Foundation of China (NSFC) (61505011); Villum Fonden (19158); H2020 European Research Council (ERC) (771878); Danmarks Grundforskningsfond (DNRF) (DNRF123).

Acknowledgment. The authors gratefully acknowledge Dr. Liang Du and Dr. Hao Hu for discussions about this Letter.

\section{REFERENCES}

1. A. J. Lowery, J. Schröder, and L. B. Du, Opt. Express 22, 1045 (2014).

2. Y. K. Huang, E. Ip, Z. Wang, M. Huang, Y. Shao, and T. Wang, J. Lightwave Technol. 29, 3838 (2011).

3. J. Du and C. Shu, J. Lightwave Technol. 31, 2307 (2013).

4. S. Shimiz, G. Gabriella, and W. Naoya, Opt. Express 20, B525 (2012).

5. D. Hillerkuss, R. Schmogrow, T. Schellinger, M. Jordan, M. Winter, G. Huber, T. Vallaitis, R. Bonk, P. Kleinow, F. Frey, M. Roeger, S. Koenig, A. Ludwig, A. Marculescu, J. Li, M. Hoh, M. Dreschmann, J. Meyer, S. B. Ezra, N. Narkiss, B. Nebendahl, F. Parmigiani, P. Petropoulos, B. Resan, A. Oehler, K. Weingarten, T. Ellermeyer, J. Lutz, M. Moeller, M. Huebner, J. Becker, C. Koos, W. Freude, and J. Leuthold, Nat. Photonics 5, 364 (2011).

6. A. J. Lowery, Opt. Express 18, 14129 (2010).

7. G. Cincotti, Opt. Lett. 36, 2321 (2011).

8. I. Kang, M. Rasras, X. Liu, S. Chandrasekhar, M. Cappuzzo, L. T. Gomez, Y. F. Chen, L. Buhl, S. Cabot, and J. Jaques, Opt. Express 19, 9111 (2011)

9. E. Palushani, H. C. Hansen Mulvad, D. Kong, P. Guan, M. Galili, and L. K. Oxenløwe, Opt. Express 22, 136 (2014).

10. Z. Wang, K. S. Kravtsov, Y. K. Huang, and P. R. Prucnal, Opt. Express 19, 4501 (2011)

11. W. Bogaerts, F. Martin, and D. Pieter, IEEE J. Sel. Top. Quantum Electron. 20, 1 (2014).

12. P. Guan, T. Hirano, K. Harako, Y. Tomiyama, T. Hirooka, and M. Nakazawa, Opt. Express 19, B567 (2011).

13. P. Guan, D. Kong, K. M. Røge, H. C. HansenMulvad, M. Galili, and L. K. Oxenløwe, Optical Fiber Communication Conference (2014), p. W4F.1.

14. E. J. M. Verdurmen, G. D. Khoe, A. M. J. Koonen, and H. D. Waardt, Microw. Opt. Technol. Lett. 48, 992 (2006).

15. P. Guan, K. M. Røge, H. C. H. Mulvad, M. Galili, H. Hu, M. Lillieholm, T. Morioka, and L. K. Oxenløwe, J. Lightwave Technol. 34, 626 (2016).

16. K. Harako, D. O. Otuya, K. Kasai, T. Hirooka, and M. Nakazawa, Opt. Express 22, 29456 (2014).

17. T. Hirooka, D. Seya, K. Harako, D. Suzuki, and M. Nakazawa, Opt. Express 23, 20858 (2015).

18. J. Azaña and M. A. Muriel, IEEE J. Quantum Electron. 36, 517 (2000).

19. Z. Geng, B. Corcoran, C. Zhu, and A. Lowery, Opt. Express 23, 29788 (2015).

20. M. Lillieholm, P. Guan, M. Galili, M. S. Møller-Kristensen, L. GrünerNielsen, and L. K. Oxenløwe, Opt. Express 25, 12566 (2017).

21. A. T. Strasser and L. W. Jefferson, IEEE J. Sel. Top. Quantum Electron. 16, 1150 (2010).

22. S. Tibuleac and M. Filer, J. Lightwave Technol. 28, 557 (2010).

23. D. Kong, J. Zang, M. Yu, Y. Li, S. Zhou, H. Guo, and J. Wu, Optical Fiber Communication Conference (2015), p. W3C.4.

24. J. Stroud and M. Foster, Opt. Express 25, 33250 (2017). 